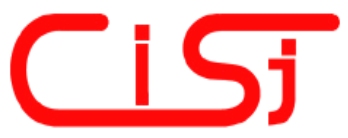

computing@tanet.edu.te.ua www.tanet.edu.te.ua/computing
ISSN 1727-6209

International Scientific Journal of Computing

\title{
COMPUTING OF LYAPUNOV EXPONENTS TECHNIQUES USING NEURAL NETWORKS
}

\author{
Vladimir Golovko ${ }^{1)}$, Yury Savitsky ${ }^{2)}$ \\ 1) Professor, Brest State Technical University, Moscowskaja 267, 224017, Brest, Belarus, gva@bstu.by \\ ${ }^{2)}$ Associate Professor, Brest State Technical University, Moscowskaja 267, 224017, Brest, Belarus, sjv@bstu.by
}

\begin{abstract}
The authors examine neural network techniques for computing of Lyapunov spectrum using observations from unknown dynamical system. Such an approach is based on applying of multilayer perceptron (MLP) for forecasting the next state of dynamical system from the previous one. It allows for evaluating the Lyapunov spectrum of unknown dynamical system accurately and efficiently only by using scalar time series. The results of experiments are discussed.
\end{abstract}

Keywords: Lyapunov spectrum, Multilayer Neural Networks, Chaotic processes, Dynamical system.

\section{INTRODUCTION}

The chaotic behaviour of a dynamical system has been manifested by the study of nonlinear mathematical equations and it has been observed on experimental data [1,2]. Unfortunately, in typical practical problems, we do not know the nonlinear equations that describe the underlying dynamical system of an observed process. The problem consist of identifying the chaotic behaviour and building a model that captures the important properties of the unknown system by using only experimental data. In order to determine the main properties of the model, we must estimate dynamic invariants of the underlying system, such as the correlation dimension, the Lyapunov exponents and the Kolmogorov entropy. However, in practice, the existing approaches for the estimation of the Lyapunov exponents from experimental data are characterized by computational complexity, require a large data length and applied only when we have all observations of dynamical system. Working on real world data, it is often difficult to obtain a reliable estimate with these approaches and thus their applicability is limited.

An important application of chaos theory is the analysis of EEG data for the detection and prediction of epileptic seizures. Epilepsy is one of the most serious neurological disorders, affecting $1 \%$ of the population in the world. The analysis of the EEG signals has been the subject of many rapidly growing studies [3]. The common approach is that a dynamical property of choice is estimated on EEG records prior to the onset of epileptic seizures and the change of the evolution of the estimated values, as time approaches the onset of a seizure, is taken as a predictor of a seizure. For example, by estimating the largest Lyapunov exponent, it has been shown that the complexity in the brain decreases as the time approaches the onset of epileptic seizure [4]. There are still open problems as to which characteristics can exhibit evolution indicating a forthcoming seizure, and there is ongoing investigation of techniques capable of capturing changes in the EEG signal prior to seizure [5]. In this respect, neural networks combined with the estimation of Lyapunov exponents may be an appropriate tool that can be developed to a robust method for automatic detection and prediction of abnormality in EEG data.

As was shown in $[6,7]$ the multilayer perceptron has been used successfully for the estimation of the Lyapunov exponents from scalar time series.

The rest of the paper is organized as follows. In section 2 is described the Lyapunov exponents. Section 3 and 4 present the techniques for computing the Lyapunov exponents respectively using all and single time series. Section 5 discusses the results of experiments. To end, Section 6 gives conclusions.

\section{THE LYAPUNOV EXPONENTS}

The concept of Lyapunov exponents existed before the establishment of chaos theory, and was developed to characterize the stability of linear as well as non-linear systems. The definition covers both discrete and continuous systems. A negative exponent indicates a local average rate of 


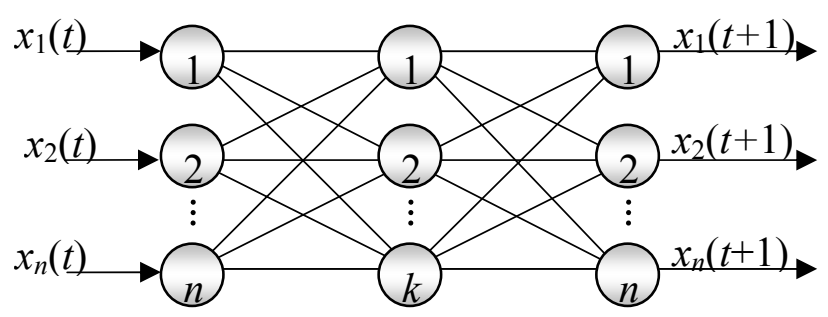

Figure 1. Predicting neural network

contraction while a positive value indicates a local average rate of expansion. Since the advent of chaos, the set or spectrum of Lyapunov exponents has been considered a measure of the effect of perturbing the initial condition of a dynamical system.

Let's consider a dynamical system described by $\mathrm{n}$ differential or difference equations. This system has $\mathrm{n}$ Lyapunov exponents $\lambda i(i=1,2, \ldots, n)$, that are globally called Lyapunov spectrum. The Lyapunov spectrum describes the system dynamics by defining the evolution of the attractor's trajectories and characterizes the sensitive dependence on the initial conditions. These exponents are the average exponential rates of convergence (divergence) of nearby trajectories in the phase space. The largest Lyapunov exponent is the statistical measure of the divergence between two orbits starting from slightly different initial conditions. In a chaotic system the largest Lyapunov exponent is positive.

Let's consider a small sphere at the initial condition in the n-dimensional phase space. Through the time this sphere is transformed into an ellipsoid with $\mathrm{n}$ principal axes: the Lyapunov spectrum measures the exponential growth for the principal axes of the evolving ellipsoid. In fact, let's consider the following Lyapunov spectrum:

$$
\lambda_{1} \geq \lambda_{2} \geq \ldots \geq \lambda_{n}
$$

and let's order the axis of the ellipsoid by decreasing length; $\lambda 1$ corresponds to the longest axis, $\lambda 2$ corresponds to the subsequent one, and so on. The Lyapunov exponent $\lambda \mathrm{i}$ is defined as:

$$
\lambda_{i}=\lim _{t \rightarrow \infty} \frac{1}{t} \cdot \ln \frac{l_{i}(t)}{l_{i}(0)}
$$

where $l_{i}(0)$ and $l_{i}(t)$ are the lengths of $i$-th axis at the initial time and at a time $t$, respectively. Therefore every Lyapunov exponent characterizes the modification of the principal axis of the ellipsoid. In an n-dimensional chaotic system the sum of the $\mathrm{n}$ Lyapunov exponents is negative for dissipative systems. The positive exponents are responsible for the sensitivity to initial conditions. The sum of the positive Lyapunov exponents is equal to Kolmogorov entropy and determines the upper prediction limit. High dimension chaotic systems tend to have very large positive exponents and predictions may be of little use.

\section{COMPUTING OF LYAPUNOV SPECTRUM USING ALL OBSERVATIONS}

Let's consider a dynamical system described by the n-dimensional observable vector $X(t)=[X 1(t), X 2(t), \ldots, X n(t)]$ and assume that the observations $\mathrm{Xi}(\mathrm{t})$ are known.

A neural network can be created to forecast the next state of dynamical system from the previous one. This network is a multilayer network with $n$ input units, $\mathrm{m}$ hidden units, and $\mathrm{n}$ output units (Fig. $1)$. The output is defined as $x(t+1)=F(x(t))$. Starting from a given initial condition, this network is able to compute the state of the dynamical system at any time, as well as to describe the evolution of the phase trajectory points. At each step the GramSchmidt orthogonalization procedure must be used to adjust the output vector.

Let $\left|w_{i}(t)\right|$ be the length of the $i$-th vector at the time $t$. This length characterizes the value of the vector along the $i$-th ellipsoid axis. Thus, the $i$-th Lyapunov's exponent is given by:

$$
\lambda_{i}=\lim _{p \rightarrow \infty} \frac{1}{p} \sum_{t=1}^{p} \ln \frac{\left|w_{i}(t)\right|}{\left|w_{i}(0)\right|}
$$

The correspondent length $\left|w_{i}(t)\right|$ can be evaluated by using a neural network and, consequently, the Lyapunov exponents can be estimated. The algorithm to compute the complete Lyapunov spectrum is as follows:

1. Take the initial point $N(0)=\left[x_{1}(0), x_{2}(0), \ldots, x_{n}(0)\right]$ from the basin of attraction.

2. Choose a small value $\varepsilon \approx 10^{-8}$ and define the coordinates of next $n$ points as follows:

$$
\begin{aligned}
& A_{1}(0)=\left[x_{1}(0)+\varepsilon, x_{2}(t), \ldots, x_{n}(t)\right] \\
& A 1(0)=[x 1(0), x 2(t)+\varepsilon, \ldots, x n(t)] \\
& \ldots \\
& A_{1}(0)=\left[x_{1}(0), x_{2}(t), \ldots, x_{n}(t)+\varepsilon\right]
\end{aligned}
$$

The following orthogonal vectors are obtained:

$$
\begin{aligned}
& N A_{1}(0)=w_{1}(0)=[\varepsilon, 0, \ldots, 0] \\
& N A 2(0)=w_{2}(0)=[0, \varepsilon, \ldots, 0] \\
& \ldots \\
& N A_{n}(0)=w_{2}(0)=[0,0, \ldots, \varepsilon]
\end{aligned}
$$


3. Compute the length of each vector $\left|N A_{i}(0)\right|=\left|w_{i}(0)\right|=\varepsilon$, where $i=\overline{1, n}$.

4. At the time $t=0$, use the set of points $N(0), A_{l}(0)$, $A_{2}(0), \ldots, A_{n}(0)$ as the input vector of the neural network. The output produced by the predicting network is the set of the coordinates of the points at the next time $t=t+1$ :

$$
\begin{aligned}
& N(1)=\left[x_{1}(1, N), x_{2}(1, N), \ldots, x_{n}(1, N)\right] \\
& A 1(1)=\left[x 1(1, A 1), x_{2}(1, A 1), \ldots, x n(1, A 1)\right] \\
& A_{2}(1)=\left[x_{1}\left(1, A_{2}\right), x_{2}\left(1, A_{2}\right), \ldots, x_{n}\left(1, A_{2}\right)\right] \\
& \ldots \\
& A_{n}(1)=\left[x_{1}\left(1, A_{n}\right), x_{2}\left(1, A_{n}\right), \ldots, x_{n}\left(1, A_{n}\right)\right],
\end{aligned}
$$

where $x_{j}\left(1, A_{j}\right)$ is the $j$-th coordinate of the point $A_{j}$ at the time $t=1$. This leads to the next set of vectors:

$$
\begin{gathered}
N A_{1}(1)=w_{1}(1)=\left[w_{11}, w_{21}, \ldots, w_{n 1}\right] \\
N A_{2}(1)=w_{2}(1)=\left[w_{12}, w_{22}, \ldots, w_{n 2}\right] \\
\ldots \\
N A_{n}(1)=w_{n}(1)=\left[w_{1 n}, w_{2 n}, \ldots, w_{n n}\right]
\end{gathered}
$$

where $w_{i j}$ is the $i$-th coordinate of the $j$-th vector, having defined $w_{i j}=x_{i}\left(1, A_{j}\right)-x_{i}(1, N)$.

5. The basis $\left[w_{1}(1), w_{2}(1), \ldots, w_{n}(1)\right]$ is transformed into the orthonormal frame by using the GramSchmidt algorithm, as follows:

a) The first vector of the orthonormal frame is chosen as:

$$
w_{1}^{\prime}(1)=\left[\frac{w_{11}}{\left|w_{1}(1)\right|}, \frac{w_{21}}{\left|w_{1}(1)\right|}, \ldots, \frac{w_{n 1}}{\left|w_{1}(1)\right|}\right]
$$

where $\left|w_{1}(1)\right|=\sqrt{w_{11}^{2}+w_{21}^{2}+\ldots+w_{n 1}^{2}}$.

b) The subsequent vectors are defined by the following recurrent formulas:

$$
\begin{gathered}
w_{i}(1)=w_{i}(1)-\sum_{j=1}^{i-1}\left(w_{i}^{T}(1) \cdot w_{j}^{\prime}(1)\right) \cdot w_{j}^{\prime}(1) \\
\left|w_{i}(1)\right|=\sqrt{w_{1 i}^{2}+w_{2 i}^{2}+\ldots+w_{n i}^{2}} \\
w_{i}^{\prime}(1)=\left[\frac{w_{1 i}}{\left|w_{i}(1)\right|}, \frac{w_{2 i}}{\left|w_{i}(1)\right|}, \ldots, \frac{w_{n i}}{\left|w_{i}(1)\right|}\right]
\end{gathered}
$$

where $i=\overline{2, n}$.

c) Compute:

$$
s_{i}(1)=\ln \frac{\left|w_{i}(1)\right|}{\left|w_{i}(0)\right|}
$$

where $i=\overline{1, n}$.

The result is the new set of points:

$$
\begin{aligned}
& N(1)=\left[x_{1}(1, N), x_{2}(1, N), \ldots, x_{n}(1, N)\right] \\
& A_{1}(1)=\left[\bar{x}_{1}\left(1, A_{1}\right), \bar{x}_{2}\left(1, A_{1}\right), \ldots, \bar{x}_{n}\left(1, A_{1}\right)\right] \\
& A_{2}(1)=\left[\bar{x}_{1}\left(1, A_{2}\right), \bar{x}_{2}\left(1, A_{2}\right), \ldots, \bar{x}_{n}\left(1, A_{2}\right)\right]
\end{aligned}
$$

$$
A_{n}(1)=\left[\bar{x}_{1}\left(1, A_{n}\right), \bar{x}_{2}\left(1, A_{n}\right), \ldots, \bar{x}_{n}\left(1, A_{n}\right)\right],
$$

where $\bar{x}_{j}\left(1, A_{j}\right)=\varepsilon \cdot w_{i j}^{\prime}+x_{i}(1, N)$.

6. Repeat from step 3 to step 5 for $t=\overline{1, p}$, where $p \approx 1000$.

7. Define the Lyapunov spectrum as:

$$
\lambda_{i}=\frac{1}{p} \sum_{t=1}^{p} s_{i}(t)
$$

where $i=\overline{1, n}$. The following Lyapunov's exponents are therefore obtained:

$$
\lambda_{1} \geq \lambda_{2} \geq \ldots \geq \lambda_{n}
$$

By using this approach, the Lyapunov exponents of the Henon time series are 0.442 and -1.625 (the actual values are 0.418 and -1.622 , respectively). For the Lorenz time series they are $0.777,0.003$, and 14.472 (the actual values are $0.906,0$, and -14.472 , respectively). Figg. 2 and 3 show the dependence of $\lambda_{i}$ from $\mathrm{p}$ for the Henon and the Lorenz time series, respectively.

\section{COMPUTING OF LYAPUNOV SPECTRUM USING SINGLE TIME SERIES}

In this case the Lyapunov spectrum can be computed by a similar way. However before we must perform the reconstruction of attractor dynamics. Let's assume now, that only one observation $X i(t)$ is known. The main goal is to compute Lyapunov exponents of unknown dynamical system using only one observation. Then the first step of proposed approach is to reconstruct the attractor dynamics from a single time series, using the method of delays [2].

After this step we can obtain the reconstructed trajectory $X(t)$, which can be presented as a matrix where each row is a phase-space vector:

$$
\mathrm{X}=[\mathrm{X}(1) \mathrm{X}(2) \ldots \mathrm{X}(\mathrm{k})] \text {, }
$$

where $X(i)$ is the state of the system at discrete time $i$ and each $X(i)$ is given by

$$
\begin{aligned}
& X(i)=[x(i) x(i-\tau) \ldots x(i-(m-1) \cdot \tau)] \\
& =\left[x_{1}(i) x_{2}(i) \ldots x_{m}(i)\right],
\end{aligned}
$$

where $\tau$ is the time delay and $m$ is the embedding dimension.

It is based on the Taken's theorem [8], which states that the attractor can be reconstructed from a one dimensional observation in a phase space with dimension $m \geq 2[d]+1$, where $d$ is the fractal dimension of the attractor and [.] is the integer part. Chaotic systems have strange attractor characterized by a non-integer dimention $d$. To apply the embedding theorem it is necessary to estimate the embedding dimension, i.e. the dimension of the reconstructed state space $m$, and the time delay, 
which is the time separation of lagged samples comprising the reconstructed state vector.

There exist several methods for the estimation of these parameters, e.g. mutual information for the delay time, false nearest neighbors and saturation of measures such as correlation dimension for the embedding dimension $[2,6]$.

The second step of proposed approach is to create neural network in order to forecast the next state of dynamical system $X(i)$ from the previous one $X(i-1)$. This network is a multilayer perceptron with $m$ input units, $k$ hidden units, and $n$ output units (Fig.1).

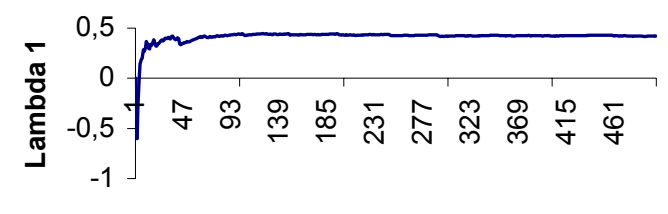

p

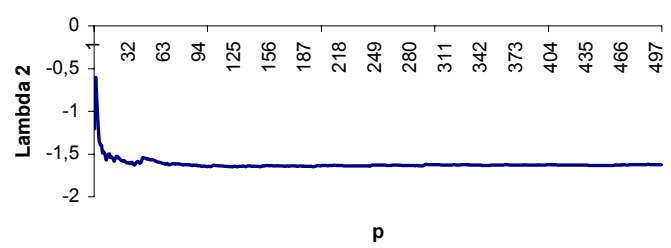

Figure 2. Estimation of the Lyapunov spectrum for the Henon time series
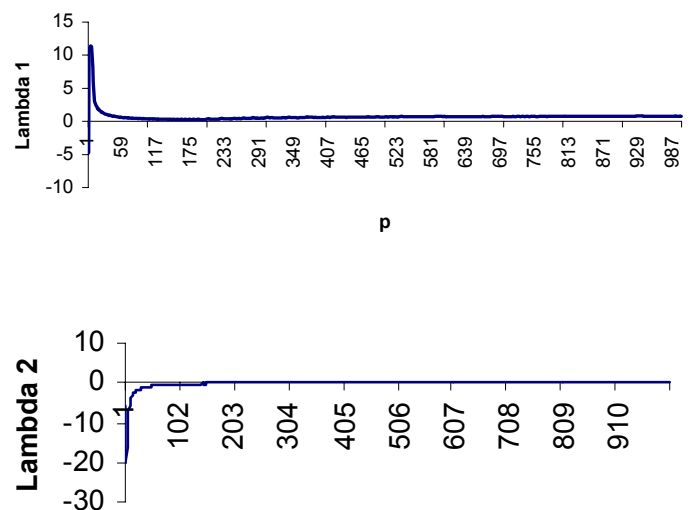

p

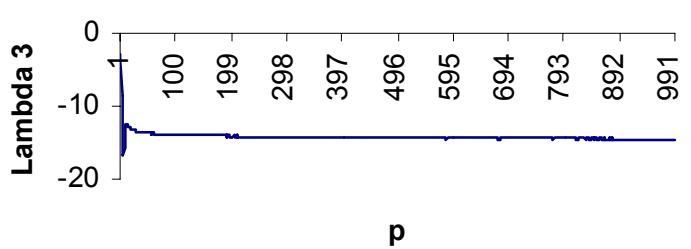

Figure 3. Estimation of the Lyapunov spectrum for the Lorenz time series
The output is defined as

$$
X(t+1)=F(X(t)) .
$$

After training neural network and starting from a given initial condition, this network is able to compute the state of the dynamical system at any time, as well as to describe the evolution of the phase trajectory points. At each step the GramSchmidt orthogonalization procedure must be used to adjust the output vector as it is shown in section 3 . Thus the proposed approach permits to estimate Lyapunov exponents using only single time series.

\section{EXPERIMENTAL RESULTS}

Let's examine proposed approach for estimation of Lyapunov spectrum. As the chaotic systems, which we want to model are the Lorenz and Roessler attractors. The Lorenz attractor is described by the following three coupled nonlinear differential equations:

$$
\left\{\begin{array}{c}
\frac{d x}{d t}=G(y-x) \\
\frac{d y}{d t}=-x z+r x-y \\
\frac{d z}{d t}=x y-b z
\end{array}\right.
$$

where $G=10, r=28$, and $b=8 / 3$ for chaotic behavior. Lorenz proposed this model for the atmospheric turbulence. For such a system actual values of Lyapunov exponents are 0.906, 0, and -14,472, respectively. The value of fractal dimension is 2.06 . The Roessler attractor can be described by the following equations:

$$
\left\{\begin{array}{l}
\frac{d x}{d t}=-y-z, \\
\frac{d y}{d t}=x+a y, \\
\frac{d z}{d t}=b+(x-r) z,
\end{array}\right.
$$

where $a=b=0.2$ and $r=5.7$ for chaotic behavior. The actual values of Lyapunov exponent are $0.07,0$, and -5.39 respictevely. The value of fractal dimension is 2.03 .

Only the X-series has been used in both cases; the size of the data set was 400 points. We have been choose the embedding dimension $m=3$ less than in accordance with Takens criterion. A multilayer perceptron with 3 input units, 10 hidden units, and 3 output units has been used. 


\section{CONCLUSION}

In this paper some aspects of chaotic time series processing have been addressed, namely estimation of the Lyapunov spectrum from all observations and from the single time series. The proposed approach based on the applying of the multilayer perceptron for estimation of Lyapunov exponents. The neural network technique allow for evaluating the Lyapunov spectrum even on small data set, that

Table 1. Estimation of Lyapunov spectrum of Lorenz system using neural network

\begin{tabular}{|c|c|c|c|c|c|c|}
\hline \multirow{2}{*}{$\mathrm{dt}$} & \multirow{2}{*}{$\mathrm{T}$} & \multicolumn{3}{|c|}{ Lyapunov spectrum } & \multirow{2}{*}{$\begin{array}{l}\text { Absolute } \\
\text { error }\end{array}$} & \multirow{2}{*}{$\begin{array}{c}\text { Relative } \\
\text { error }\end{array}$} \\
\hline & & $\lambda_{1}$ & $\lambda_{2}$ & $\lambda_{3}$ & & \\
\hline 0,04170 & 0,1668 & 0,612978 & $-0,2016840$ & $-15,0033$ & 0,559053 & $3,83 \%$ \\
\hline 0,04200 & 0,1680 & 0,725777 & $-0,0211582$ & $-14,6402$ & 0,193839 & $1,33 \%$ \\
\hline 0,04215 & 0,1686 & 0,966544 & $-0,3009800$ & $-15,9458$ & 1,407730 & $9,64 \%$ \\
\hline 0,04220 & 0,1688 & 0,965399 & $-0,3006240$ & $-15,9270$ & 1,389170 & $9,51 \%$ \\
\hline 0,08500 & 0,1700 & 1,143851 & $-0,2816490$ & $-14,9843$ & 0,553092 & $3,79 \%$ \\
\hline 0,04260 & 0,1704 & 1,021790 & $-0,4326160$ & $-15,6514$ & 1,168620 & $8,00 \%$ \\
\hline 0,04260 & 0,1704 & 0,483841 & 0,0528098 & $-13,3949$ & 1,251610 & $8,57 \%$ \\
\hline 0,04300 & 0,1720 & 0,742471 & $-0,0865899$ & $-14,2650$ & 0,358420 & $2,45 \%$ \\
\hline 0,08600 & 0,1720 & 0,830438 & $-0,3357490$ & $-13,5627$ & 1,066370 & $7,30 \%$ \\
\hline 0,04320 & 0,1728 & 0,570654 & $-0,1465600$ & $-14,9297$ & 0,511766 & $3,51 \%$ \\
\hline 0,08700 & 0,1740 & 1,216890 & $-0,6435080$ & $-14,5374$ & 0,715508 & $4,90 \%$ \\
\hline
\end{tabular}

Table 2. Estimation of Lyapunov spectrum of Roessler system using neural network

\begin{tabular}{|c|c|c|c|c|c|c|}
\hline \multirow{2}{*}{$\mathrm{dt}$} & \multirow{2}{*}{$\tau$} & \multicolumn{3}{|c|}{ Lyapunov spectrum } & \multirow{2}{*}{$\begin{array}{l}\text { Absolute } \\
\text { error }\end{array}$} & \multirow{2}{*}{$\begin{array}{l}\text { Relative } \\
\text { error }\end{array}$} \\
\hline & & $\lambda_{1}$ & $\lambda_{2}$ & $\lambda_{3}$ & & \\
\hline 0,04 & 0,04 & 0,173003 & $-0,0821049$ & $-5,47571$ & 0,154879 & $2,87 \%$ \\
\hline 0,07 & 0,07 & 0,060350 & $-0,3888620$ & $-5,18352$ & 0,441825 & $8,19 \%$ \\
\hline 0,06 & 0,12 & 0,090696 & 0,0030709 & $-5,02998$ & 0,363565 & $6,74 \%$ \\
\hline 0,06 & 0,12 & 0,106080 & $-0,0358488$ & $-5,79224$ & 0,402378 & $7,46 \%$ \\
\hline 0,06 & 0,12 & 0,077922 & $-0,0187908$ & $-5,93021$ & 0,537581 & $9,97 \%$ \\
\hline 0,07 & 0,14 & 0,129117 & $-0,1092460$ & $-4,93167$ & 0,477637 & $8,86 \%$ \\
\hline 0,08 & 0,16 & 0,106981 & $-0,0449128$ & $-5,36074$ & 0,065971 & $1,22 \%$ \\
\hline 0,08 & 0,16 & 0,085461 & $-0,0282390$ & $-5,31476$ & 0,084427 & $1,57 \%$ \\
\hline 0,04 & 0,16 & 0,119605 & $-0,2027930$ & $-5,56896$ & 0,272851 & $5,06 \%$ \\
\hline 0,06 & 0,18 & 0,141245 & $-0,0751598$ & $-5,48983$ & 0,141277 & $2,62 \%$ \\
\hline 0,08 & 0,48 & 0,078753 & $-0,0144016$ & $-5,24691$ & 0,147000 & $2,73 \%$ \\
\hline
\end{tabular}


permits both for reducing the computationally complexity and for limit the observation time.

\section{REFERENCES}

[1] Ilya Prigogine. The End of Certainty: Time, Chaos, and the New Laws of Nature. The Free Press, N.Y., 1997, 240p.

[2] Holger Kantz, Tomas Schreiber. Nonlinear time series analysis. Cambridge, Cambridge University Press, 1997.

[3] Proceedings of the 1999 Workshop "Chaos in Brain?". World Scientific, Singapore, 2000.

[4] L. D. Iasemides, J. C. Sackellares. Chaos theory and epilepsy. The Neuroscientist, 2 (1996), pp. 118-126.

[5] D. Kugiumtzis, P. G. Larsson. "Linear and Nonlinear Analysis of EEG for the Prediction of Epileptic Seizures", Proceedings of the 1999 Workshop "Chaos in Brain?", World Scientific, Singapore, pp. 329 - 333, 2000.

[6] V. Golovko. From Neural Networks to Intelligent Systems: Selected Aspects of Training, Application and Evolution // chapter of NATO book "Limitations and Future Trends in Neural Computation". - Amsterdam: IOS Press, 2003. - 25p. [7] V. Golovko, Y. Savitsky, N. Maniakov. Neural Networks for Signal Processing in Measurement Analysis and Industrial Applications: the Case of Chaotic Signal Processing // chapter of NATO book "Neural networks for instrumentation, measurement and related industrial applications". Amsterdam: IOS Press, 2003, pp. 119-143.

[8] F. Takens. Detecting strange attractors in turbulence, Lecture Notes in Mathematics, Vol. 898, Springer-Verlag, Berlin, 1980, pp. 366-381; and in Dynamical System in Turbulence, Warlock, 1980, eds. D. Rand and L.S. Young.

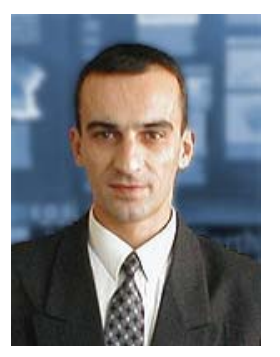

Vladimir Golovko was born in Belarus in 1960. He received M.E. degree in Computer Engineering in 1984 from the Moscow Bauman State Technical University. In 1990 he received $P h D$ degree from the Belarus State University and in 2003 he received doctor science degree in Computer Science from the United Institute of Informatics problems national Academy of Sciences (Belarus). At present he work as head of Intelligence Information Technologies Department and Laboratory of Artificial Neural Networks of the Brest State Technical University. His research interests include Artificial Intelligence, neural networks, autonomous learning robot, signal processing, chaotic processes, intrusion and epilepsy detection. He has published more than 150 scientific papers, including 3 books and 2 chapters of books.

Yury Savitsky was born in Belarus in 1971. He received M.E. degree with honors in Computer Engineering in 1993 from the Brest Polytechnic Institute, Belarus. In 2000 he received $P h D$ degree from the Belarusian State University of Informatics and Radioelectronics, Minsk, Belarus. At present he is associate professor of Intelligence Information Technologies Department and member of Laboratory of Artificial Neural Networks of the Brest State Technical University. His research interests include Artificial Intelligence, neural network technique for time series prediction, signal processing, chaotic data analysis. He has published more than 50 scientific papers, including 1 chapter of book. 